\title{
QUANTITATIVE ANALYSIS OF DYSTROPHIN IN HUMAN AND RODENT MUSCLES
}

\author{
Ritsuko Koga ${ }^{1}$, Shoichi Ishiura ${ }^{1}$, Kitchi Arahata ${ }^{1}$, Toshifumi Takakuwa ${ }^{2}$, IkuYa Nonaka ${ }^{1}$ and \\ Hideo Sugita ${ }^{1}$ \\ ${ }^{1}$ National Institute of Neuroscience, NCNP, Kodaira, Tokyo 187, and ${ }^{2}$ Department of Pathology, St. Marianna University School of \\ Medicine, Kawasaki 216, Japan
}

\begin{abstract}
A method for the quantitative analysis of dystrophin using an immunochemical technique is described. The method is applicable to measure the dystrophin content in various human and rodent muscles. There is no significant difference in the dystrophin content between the soleus and extensor digitorum longus muscles of rodents, nor among 8 different human muscles. The result suggests that the vulnerability of skeletal muscle involved in Duchenne muscular dystrophy does not depend on the dystrophin content in each muscle.
\end{abstract}

Dystrophin is a protein product of the Duchenne muscular dystrophy (DMD) gene, and sequence analysis predicts a $427 \mathrm{kDa}$ protein composed of 3,685 amino acids $(5,7,8,10,11,14)$. Dystrophin is thought to be a membrane-associated cytoskeletal component of muscle fibers in normal skeletal and cardiac muscles, but is absent in muscles from DMD patients $(1-3,16,18,20)$. Although the function of dystrophin has not yet been fully clarified, the absence of dystrophin is currently believed to be the primary cause of muscle fiber degeneration in DMD. Further investigation of the biochemical properties of dystrophin seems necessary for a better understanding of its biological function.

Recently there have been some reports describing the dystrophin content of human muscle biopsy specimens and some rodent muscles and cultured muscle cells $(4,9)$. In the present study, we describe a method for the immunochemical quantification of dystrophin by densitometry in various kinds of human and rodent skeletal muscles. The relative amount of dystrophin in rodent soleus and extensor digitorum longus (EDL) muscles is almost the same, and 8 different kinds of human muscles also contain a nearly constant amount of dystrophin.

\section{MATERIALS AND METHODS}

\section{Antiserum}

Antiserum was produced in rabbits through immu- nization with a synthetic peptide consisting of 50 amino acids from the $\mathrm{C}$-terminus of dystrophin (1).

\section{Sample Preparation}

The human autopsy muscles were obtained from an adult cadaver free from neuromuscular diseases $4 \mathrm{~h}$ after death. Muscle biopsy specimens were obtained from patients undergoing the procedure for diagnostic purposes with informed consent.

Approximately 5-10 mg of muscle were homogenized by sonication with $20 \mathrm{vol}$ of sample buffer (0.125 M Tris-HC1 buffer, $\mathrm{pH}$ 6.8, 5\% 2-mercaptoethanol, $2 \%$ SDS, $10 \%$ glycerol and $0.1 \%$ bromophenolblue). After incubation at $100^{\circ} \mathrm{C}$ for $5 \mathrm{~min}$, insoluble particles were removed by centrifugation at $15,000 \mathrm{rpm}$ for $1 \mathrm{~min}$.

\section{Quantitative Analysis of Myosin}

To obtain the same amount of muscle tissue per sample, all biopsy samples were first subjected to quantification of their myosin content. As a control, normal muscle was prepared by the same procedure. Samples, 1, 2 and $5 \mu 1$, were electrophoresed in 6\% SDS-polyacrylamide gels $(1 \mathrm{~mm} \times$ $8 \mathrm{~cm} \times 6 \mathrm{~cm} ; 12$ lanes) with a $3.5 \%$ stacking gel, according to Laemmli (12). Gels were run at $8 \mathrm{~mA}$ for the stacking gel and $16 \mathrm{~mA}$ for the separating 
gel until the dye front reached the bottom of the gel. After electrophoresis, the gels were stained with Coomassie brilliant blue $(0.2 \%$ in $50 \%$ methanol and 10\% acetic acid), and destained with 7\% acetic acid. The gels were then subjected to densitometry, and the optical density of the myosin heavy chain was measured with an ACI image analysis system (ACI Japan).

\section{Western Blotting Analysis}

Samples, each containing the same amount of myosin, were electrophoresed on a $6 \%$ SDS-polyacrylamide gel $(1 \mathrm{~mm} \times 16 \mathrm{~cm} \times 16 \mathrm{~cm})$. After electrophoresis, the gels were transferred to a nitrocellulose membrane (17) at $50 \mathrm{~mA}$ overnight at $4^{\circ} \mathrm{C}$ in transfer buffer (50 mM Tris-glycine buffer, $\mathrm{pH} 8.7$, $20 \%$ ethanol and $0.1 \%$ SDS). After transfer, the membrane was rinsed briefly with TPBS $(25 \mathrm{mM}$ phosphate buffer, $\mathrm{pH} 7.4,0.5 \mathrm{M} \mathrm{NaCl}$ and $0.02 \%$ Tween-20) to remove ethanol and SDS in the transfer buffer. The membrane was blocked for $3 \mathrm{~h}$ at room temperature with 5\% skimmed milk in TPBS and another $3 \mathrm{~h}$ at room temperature with 20\% normal goat serum in TPBS. The membrane was then incubated overnight at $4^{\circ} \mathrm{C}$ with antiserum diluted 1:200 with 20\% normal goat serum. The membrane was washed with TPBS ( $7 \mathrm{~min}, 3$ times) and incubated with a biotinylated goat anti-rabbit secondary antibody for $60 \mathrm{~min}$ at room temperature. After washing with TPBS (7 min, 3 times), the membrane was incubated with $0.6 \%$ hydrogen peroxide in $20 \%$ methanol for $30 \mathrm{~min}$ at room temperature to block the endogenous peroxidase. The membrane was washed with TPBS $(7 \mathrm{~min}$, 3 times), incubated with avidin-peroxidase for $60 \mathrm{~min}$ at room temperature, washed with TPBS ( $7 \mathrm{~min}, 3$ times), and rinsed with PBS (without Tween-20) to remove Tween-20, an inhibitor of the peroxidase reaction. The membrane was developed with 4-chloro-1-naphthol and hydrogen peroxide (3 $\mathrm{mg}$ of 4-chloro-1-naphthol in $2 \mathrm{ml}$ methanol, $10 \mathrm{ml}$ PBS and $5 \mu 1$ hydrogen peroxide), washed with distilled water, air-dried, and subjected to densitometry. The optical density of the immunoreactive dystrophin bands was measured and the relative values of the samples were calculated as a percentage of the optical density of the control.

\section{RESULTS}

The standard curve was prepared using normal human biceps brachii muscle. The optical density of the immunoreactive dystrophin band was linearly related to the amount of human muscle up to $50 \mu \mathrm{g}$ (Fig. 1).

Using this technique, the dystrophin content in various kinds of human and rodent muscles were analyzed. Fig. 2 shows a comparison of the dystrophin content of soleus and EDL muscles in rats $(\mathrm{R})$, mice $(\mathrm{M})$ and guinea pigs $(\mathrm{G})$. The ratio of the dystrophin content of soleus (S) and EDL (E) is nearly 1 . The results suggest that the dystrophin content of slow muscle is almost identical to that of fast muscle.

Among 8 different human muscles (Fig. 3), the dystrophin content of pharyngeal muscle was slightly higher than in other muscles. In limb muscles, there is no difference between proximal (biceps brachii, rectus femoris) and distal (abductor pollicis, gastrocnemius) muscles in both the upper and lower extremities. The dystrophin content of the diaphragm and heart muscle is essentially the same as that in limb muscles. Fig. 4 shows the results of a typical routine analysis. Muscles obtained by biopsy from DMD and Becker muscular dystrophy (BMD) patients were analyzed along with normal muscle (lanes 2, 5). No dystrophin was detected in DMD muscle (lane 1), while lower amounts of dystrophin with reduced molecular weights were observed in BMD muscles (lanes 3, 4).

\section{DISCUSSION}

Methods for the quantitative analysis of dystrophin have been reported recently $(4,9)$. As the amount of dystrophin in muscle is very low (about $0.002 \%$ of total muscle protein), Western blotting is the only way to detect dystrophin. Here the specificity and activity of the antibody is critical to the analytical outcome. Additionally, the experimental conditions should be adjusted for each antibody used. The transfer step (overnight at low temperature, with $0.1 \%$ SDS and ethanol), and double blocking, overnight incubation at $4^{\circ} \mathrm{C}$ with diluted antibody, are essential to obtain a clear dystrophin band with low background. The method described in this paper has been used routinely as a method for dystrophin analysis in our laboratory, and gives reliable and reproducible results on the dystrophin content and molecular weight (Fig. 4).

Using this method, the amount of dystrophin in various muscles was compared. We have found in this study that there are no differences in the content of dystrophin in soleus and EDL muscles, or 
A

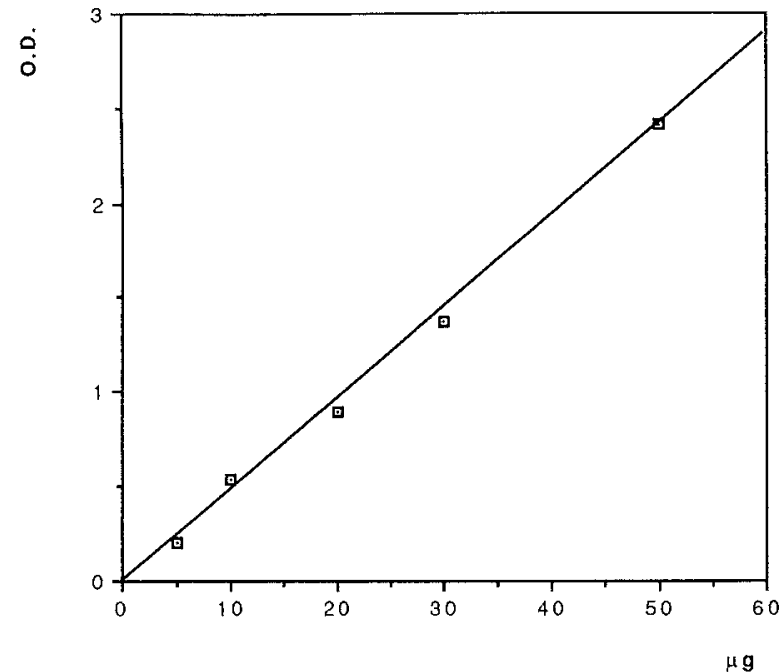

B

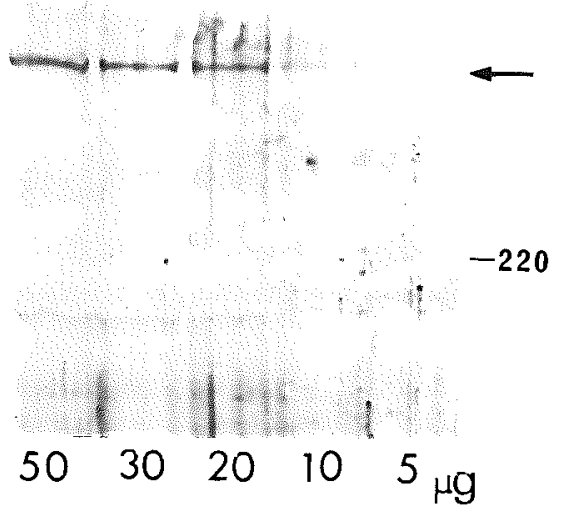

Fig. 1 Immunochemical detection of dystrophin in normal human biceps muscle. A: The relationship between the optical density of the dystrophin band and the total amount of muscle applied per well. B: Dystrophin immunoblots using $50 \mu \mathrm{g}, 30 \mu \mathrm{g}, 20 \mu \mathrm{g}, 10 \mu \mathrm{g}$ and $5 \mu \mathrm{g}$ of human muscle. The position of dystrophin (arrow) and a molecular weight marker (220 kDa: myosin heavy chain) is indicated.

M

$\mathbf{R}$

G

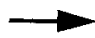

$+\infty+\infty+\infty$

220

\section{$\begin{array}{llllll}S & E & S & E & S & E\end{array}$}

Fig. 2 Comparison of the dystrophin content of rodent soleus (S) and EDL (E) muscles. Representative dystrophin immunoblots of mouse (M), rat (R) and guinea-pig $(\mathrm{G})$ muscles are shown (arrow). The soleus/ EDL ratios of the optical density of dystrophin (the mean of four independent experiments) are 1.05 in mice, 1.11 in rats and 1.12 in guinea pigs. Molecular weight markers are myosin heavy chain $(220 \mathrm{kDa})$ and phosphorylase $b(94 \mathrm{kDa})$. 


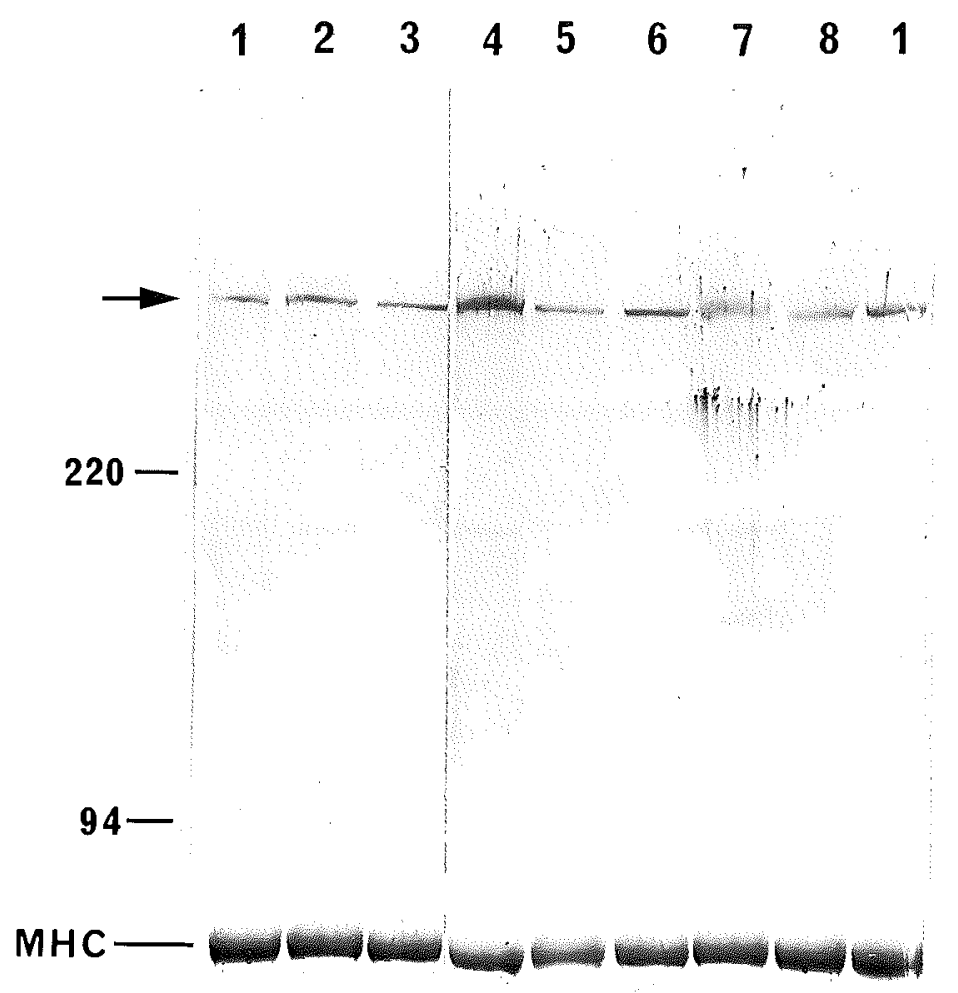

Fig. 3 The dystrophin content in 8 different human muscles. Dystrophin immunoblot (arrow), and the myosin heavy chain (MHC) stained with Coomassie brilliant blue are shown. The ratio of the optical density of dystrophin is: rectus femoris (lane 1), 1.00; biceps brachii (lane 2), 1.05; abductor pollicis (lane 3), 0.96; pharyngeal muscle (lane 4), 1.23; intercostal muscle (lane 5), 1.03; diaphragm (lane 6), 1.13; heart (lane 7), 0.93; and gastrocnemius (lane 8), 0.94. Molecular weight markers are as in Fig. 2.

slow and fast muscles of rodents (Fig. 2). Accumulated data suggest that dystrophin is located beneath the plasma membrane as a component of the cytoskeleton where it plays an important role in the maintenance of the mechanical integrity during contraction and relaxation $(6,7,15)$. In this context, it is very interesting to note that the dystrophin content of various types of skeletal muscle cell is almost the same in spite of their variable locations. It is necessary to increase the number of observations before we conclude that a slightly higher dystrophin content in pharyngeal muscle, which is innervated by the cranial nerve, is a meaningful finding (Fig. 3). There are reports $(13,19)$ that in Duchenne muscular dystrophy, the white glycolytic muscle tends to be digested faster than the red oxidative muscle. To clarify the difference in the susceptibility of dystrophin-deficient muscles to degradation, we first analyzed the dys- trophin content of various human muscles. Contrary to our expectation, there was no significant difference between proximal and distal parts of limb muscles, diaphragm, pharyngeal muscle, or heart muscle. The results strongly suggest that the susceptibility of muscle proteins to degradation in dystrophin-deficient DMD muscles can not be explained by the amount of dystrophin, but is related to some other mechanisms in respective muscles.

Received 23 March 1992; and accepted 20 April 1992

\section{REFERENCES}

1. Arahata K., Ishiura $\mathrm{S}$., Ishiguro $\mathrm{T}$., Tsukahara $\mathrm{T}$., Suhara Y., Eguchi C., Ishihara T., Nonaka I., Ozawa E. and Sugrta H. (1988) Immunostaining of skeletal and cardiac muscle surface membrane with antibody against Duchenne muscular dystrophy peptide. Nature 333, 861863 


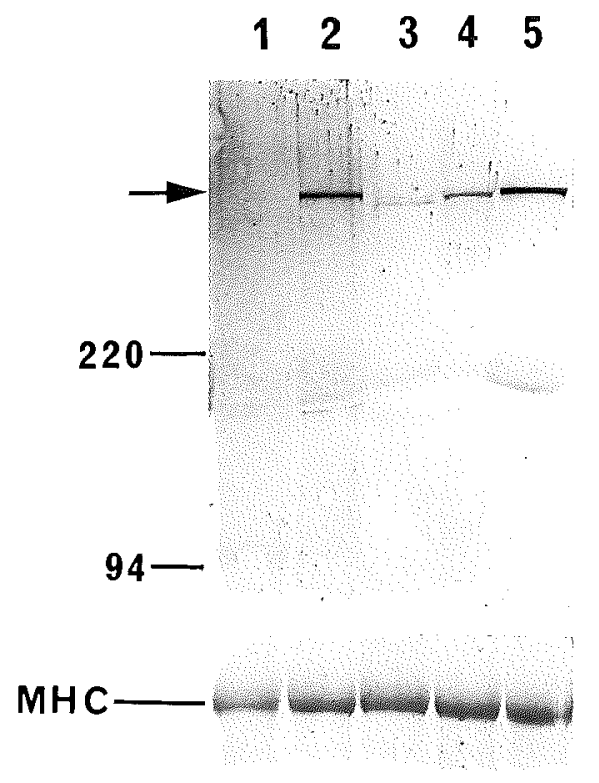

Fig. 4 Analysis of the dystrophin content of human muscles obtained by biopsy. Normal (lanes 2, 5), DMD (lane 1) and BMD (lanes 3, 4) muscles were electrophoresed and immunoblotted, and $\mathrm{MHC}$ were stained with Coomassie brilliant blue. In DMD muscle (lane 1) dystrophin was not detected. In BMD muscles, the dystrophin content was 14\% (lane 3) and 29\% (lane 4) of that in normal muscle $(100 \%)$. In BMD muscles, changes in the molecular weight of dystrophin were detected, i.e. $360 \mathrm{kDa}$ in lane 3 and $390 \mathrm{kDa}$ in lane 4 . The molecular weight was calculated based on the mobility of normal dystrophin $(427 \mathrm{kDa})$ and myosin heavy chain $(220 \mathrm{kDa})$. The arrow indicates normal dystrophin, and molecular weight markers are as in Fig. 2.

2. Arahata K., Ishiura S., Tsukahara T, and Sugita H. (1989) Dystrophin digest. Nature 337, 606

3. Bonilla E., Samitt C. E., Miranda A. F, Hays A. P., Salviati G., DiMauro S., Kunkel L. M., Hoffman E. P. and ROWLAND L. P. (1988) Duchenne muscular dystrophy: Deficiency of dystrophin at the muscle cell surface. Cell 54, 447-452

4. Bulman D. E., Murphy E. G., Zubrzycka-GaArn E. E., WORTON R. G. and RAY P. N. (1991) Differentiation of Duchenne and Becker muscular dystrophy phenotypes with amino- and carboxy-terminal antisera specific for dystrophin. Amer. J. Hum. Genet. 48, 295-304

5. Burghes A. H. M., Logan C., Hu X., Belfall B., Worton R. G. and RAY P. N. (1987) A cDNA clone from the Duchenne/Becker muscular dystrophy gene. Nature 328, 434-437

6. Ervasti J. M. and Campbell K. P. (1991) Membrane organization of the dystrophin-glycoprotein complex. Cell 66, 1121-1131

7. Hoffman E. P., Brown R. H. and Kunkel L. M. (1987) Dystrophin: The protein product of the Duchenne muscular dystrophy locus. Cell 51, 919-928

8. Hoffman E. P., Knudson C. M., Campbell K. P. and Kunkel L. M. (1987) Subcellular fractionation of dystrophin to the triads of skeletal muscle. Nature 330, 754-758

9. Ho-Kim M. A., Bédard A., Vincent M. and Rogers P. A. (1991) Dystrophin: A sensitive and reliable immunochemical assay in tissue and cell culture homogenates. Biochem. Biophys. Res. Commun. 181, 1164-1172

10. Koenig M., Hoffman E. P., Bertelson C. J., Monaco A. P., Feener C. and Kunkel L. M. (1987) Complete cloning of the Duchenne muscular dystrophy (DMD) cDNA and preliminary genomic organization of the DMD gene in normal and affected individuals. Cell 50, 509-517

11. Koenig M., Monaco A. P. and Kunkel L. M. (1988) The complete sequence of dystrophin predicts a rod-shaped cytoskeletal protein. Cell 53, 219-228

12. LaEmmLi U. K. (1970) Cleavage of structural proteins during the assembly of the head of bacteriophage T4. Nature 227, 680-685

13. Minetti C., Ricci E. and Bonilla E. (1991) Progressive depletion of fast alpha-actinin-positive muscle fibers in Duchenne muscular dystrophy. Neurology 41, 1977-1981

14. Monaco A. P., Neve R. L., Colletti-Feener C., BertelSON C. J., KuRNit D. M. and KunKel L. M. (1986) Isolation of candidate cDNAs for portions of the Duchenne muscular dystrophy gene. Nature 323, 646-650

15. Récan D., Chafey P., Leturce F., Hugnot J.-P., Vincent N., Tomé F., Collin H., Simon D., CZernichow P., Nicholson L. V. B., FardeaU M., Kaplan J.-C. and Chelly J. (1992) Are cysteine-rich and COOH-terminal domains of dystrophin critical for sarcolemmal localization? J. Clin. Invest. 89, 712-716

16. Sugita H., Arahata K., Ishiguro T., Suhara Y., Tsukahara T., Ishiura S., Eguchi C., Nonaka I. and Ozawa E. (1988) Negative immunostaining of Duchenne muscular dystrophy (DMD) and mdx mouse muscle surface membrane with antibody against synthetic peptide fragment predicted from DMD cDNA. Proc. Jap. Acad. 64, Ser. B, 37-39

17. Towbin H., Staehelin T. and Gordon J. (1979) Electrophoretic transfer of proteins from polyacrylamide gels to nitrocellulose sheets: Procedure and some applications. Proc. Natl. Acad. Sci. USA 76, 4350-4354

18. Watkins S. C., Hoffman E. P., Slayter H. S. and Kunkel L. M. (1988) Immunoelectron microscopic localization of dystrophin in myofibres. Nature 333, 863-866

19. Webster C., Silberstein L., Hays A. P. and Blau H. M. (1988) Fast muscle fibers are preferentially affected in Duchenne muscular dystrophy. Cell 52, 503-513

20. Zubrzycha-GaARn E. E., Bulman D. E., Karpati G., Burghes A. H. M., Belfall B., Klamut H. J., Talbot J., Hodges R. S., Ray P. N. and Worton R. G. (1988) The Duchenne muscular dystrophy gene product is localized in sarcolemma of human skeletal muscle. Nature 333, 466469 